\title{
Phototherapeutic keratectomy for epithelial basement membrane dystrophy
}

\author{
This article was published in the following Dove Press journal: \\ Clinical Ophthalmology \\ 16 December 2016 \\ Number of times this article has been viewed
}

\section{Wen-Shin Lee \\ Carson K Lam \\ Edward E Manche}

Department of Ophthalmology, Stanford University, Stanford, CA, USA
Correspondence: Edward E Manche Department of Ophthalmology, Stanford University, 2452 Watson Court, Stanford, CA 94303, USA

Tel +l 6507236995

Fax + I 6503209443

Email edward.manche@stanford.edu
Purpose: The purpose of this study was to evaluate the long-term efficacy of phototherapeutic keratectomy (PTK) in treating epithelial basement membrane dystrophy (EBMD).

Methods: Preoperative and postoperative records were reviewed for 58 eyes of 51 patients with $>3$ months follow-up (range 3-170 months) treated for EBMD with PTK after failure of conservative medical treatment at Byers Eye Institute of Stanford University. Symptoms, clinical findings, and corrected distance visual acuity (CDVA) were assessed. The primary outcome measure was symptomatic recurrence as measured by erosions or visual complaints $>3$ months after successful PTK.

Results: For eyes with visual disturbances ( $n=30$ ), preoperative CDVA was $~ 20 / 32$ (0.24 LogMAR, SD 0.21) and postoperative CDVA was $~ 20 / 25$ (0.07 LogMAR, SD 0.12; $P<0.0001)$. Twenty-six eyes $(86.7 \%)$ responded to treatment, with symptomatic recurrence in 6 eyes $(23.1 \%)$ at an average of 37.7 months (SD 42.8). For eyes with painful erosions $(n=29)$, preoperative CDVA was $20 / 25$ (0.12, SD 0.19) and postoperative CDVA was $20 / 20$ (0.05. SD 0.16; $P=0.0785)$. Twenty-three eyes $(79.3 \%)$ responded to treatment, with symptomatic recurrence in 3 eyes (13.0\%) at an average of 9.7 months (SD 1.5). The probability of being recurrence free after a successful treatment for visual disturbances and erosions at 5 years postoperatively was estimated at $83.0 \%$ (95\% confidence interval 68.7\%-97.0\%) and 88.0\% (95\% confidence interval $65.3 \%-96.6 \%$ ), respectively.

Conclusion: The majority of visual disturbances and painful erosions associated with EBMD respond to PTK. For those with a treatment response, symptomatic relief is maintained over long-term follow-up.

Keywords: epithelial basement membrane dystrophy, EBMD, phototherapeutic keratectomy, PTK

\section{Introduction}

In the last 2 decades, the use of excimer laser phototherapeutic keratectomy (PTK) has become an accepted modality for the treatment of several anterior corneal irregularities, including persistent epithelial defects and superficial opacities of the cornea. ${ }^{1,2}$ Epithelial basement membrane dystrophy (EBMD) is commonly treated with PTK at our institution.

EBMD, also known as map-dot-fingerprint, anterior basement membrane, or Cogan's microcystic epithelial dystrophy, is a common dystrophy, with $>2 \%$ of the population estimated to be affected. EBMD stems from an inherent dysfunction in the basal epithelial cells resulting in the secretion of abnormal basement membrane that extends into the epithelium as well as the accumulation of fibrillogranular material both between Bowman's layer and basement membrane and within the epithelium. ${ }^{3}$ It is characterized by epithelial microcysts, map-like configurations, and fingerprint 
lines as a result of irregular and redundant basement membrane proliferation.

Approximately $10 \%$ of EBMD patients experience symptoms of either pain from recurrent corneal erosions or decreased vision secondary to corneal surface irregularity. ${ }^{3,4}$ Treatment for symptomatic EBMD begins with topical lubricants, hypertonic solutions, and bandage soft contact lenses, while medically refractory cases are recommended for surgical treatments such as stromal puncture with a needle or neodymium:YAG laser and epithelial debridement. ${ }^{5,6}$ In cases of EBMD, epithelial debridement with diamond-dusted burr polishing or PTK ablation to the level of Bowman's membrane has shown to be effective. ${ }^{7-11}$

To the authors' knowledge, the present study is the largest series of EBMD eyes treated with PTK to date. We present the long-term outcomes of PTK, our preferred technique, performed on a cohort of patients who had failed conservative management for EBMD. We hypothesize that those patients with a favorable early posttreatment course continue to benefit from treatment in the long term.

\section{Methods}

\section{Study design and population}

A search of medical records to identify all patients who had undergone PTK for EBMD between September 2000 and September 2015 at the Byers Eye Institute was performed. The benefits and risks of PTK were discussed with all patients, and written informed consent was obtained. Ethical approval for this retrospective study was granted by the institutional review board of Stanford University Medical Center and was conducted in strict adherence to Health Insurance Portability and Accountability Act of 1996 and the tenets of the Declaration of Helsinki. Patients were included in the study if they had failed conservative treatment for EBMD. EBMD patients presented with symptomatic recurrent corneal erosions, irritation, or visual symptoms with morphological slit-lamp findings of EBMD. Patients were considered to have recurrent erosions if they had painful episodes at night or on awakening in conjunction with slit-lamp findings of epithelial irregularity. Eyes were considered to have morphological slit-lamp findings of EBMD if microcysts, maps, dots, or fingerprint changes were seen on slit-lamp examination.

\section{Procedure}

A PRK spatula was used to mechanically remove the corneal epithelium. All epithelium within $1 \mathrm{~mm}$ of the limbus was removed. The AMO Visx Star excimer laser (193 nm wavelength, $160 \mathrm{~mJ} / \mathrm{cm}^{2}$ fluence; Abbott Medical Optics Inc, Santa Ana, CA, USA) was used for all PTK treatments with a repetition rate of $2 \mathrm{~Hz}$. The central cornea was treated using a 6.0-mm spherical treatment zone with $\sim 5 \mu \mathrm{m}$ (15-20 pulses) of tissue removal. The peripheral cornea was treated using a spherical 3-mm zone in a donut-like pattern using the joystick and head movement to apply the treatment. Postoperative management included a bandage contact lens used along with fluorometholone (FML) 1\% twice a day and moxifloxacin or ciprofloxacin four times a day until the bandage contact lens was removed. FML was used for 2 additional weeks after the contact lens was removed. Patients were continued on Muro 128 for at least 1 month and longer as needed.

\section{Outcome measures}

Data were collected at initial visit and each follow-up appointment after PTK. The patient's corrected distance visual acuity (CDVA), symptoms, corneal findings with special attention to corneal haze and EBMD features, history of recent erosions, and manifest refraction were recorded. The presence of recurrent erosions, visual complaints or irritation with characteristic EBMD morphological features anywhere on the cornea 3 months after successful PTK, and reepithelialization were criteria for recurrence.

\section{Statistical analysis}

CDVA in Snellen acuity was converted to LogMAR for use in calculations. Postoperative CDVA to preoperative CDVA was compared with paired, 2-tailed $t$-test for normally distributed data and with Wilcoxon signed-rank test for nonnormally distributed data as determined by the Shapiro-Wilk test. Postoperative CDVA and duration of time to recurrence between subgroups were compared with 2 -variable $t$-test for normally distributed data and with Wilcoxon rank-sum test for non-normally distributed data as determined by the Shapiro-Wilk test. The correlation of CDVA stability with time was estimated by calculating the Pearson productmoment correlation coefficient. The cumulative probability of recurrence of EBMD-related symptoms was determined using the Kaplan-Meier method. Statistical analysis was performed using Stata (StataCorp LP, College Station, TX, USA) and Excel (Microsoft Corporation, Redmond, WA, USA).

\section{Results}

\section{Patient population}

Fifty-eight eyes from 51 patients were studied. All patients had complete ophthalmologic examination including CDVA 
Table I Summary of demographics and outcomes

\begin{tabular}{|c|c|c|c|c|c|c|c|c|c|c|}
\hline Group & $\mathbf{n}$ & $\begin{array}{l}\text { Age } \\
\text { (years) }\end{array}$ & $\begin{array}{l}\text { Follow-up } \\
\text { (months) }\end{array}$ & $\begin{array}{l}\text { Preoperative } \\
\text { BCVA } \\
\text { (LogMAR) }\end{array}$ & $\begin{array}{l}\text { Postoperative } \\
\text { BCVA } \\
\text { (LogMAR) }\end{array}$ & $\begin{array}{l}\text { Change } \\
\text { in BCVA } \\
\text { (LogMAR) }\end{array}$ & $\begin{array}{l}\text { Worse } \\
\text { BCVA* } \\
\text { (LogMAR) }\end{array}$ & $\begin{array}{l}\text { Primary } \\
\text { treatment } \\
\text { response, } n \text { (\%) }\end{array}$ & $\begin{array}{l}\text { Symptomatic } \\
\text { recurrence, } \\
\text { n (\%) }\end{array}$ & $\begin{array}{l}\text { Time to } \\
\text { recurrence } \\
\text { (months) }\end{array}$ \\
\hline $\begin{array}{l}\text { Visual } \\
\text { disturbances }\end{array}$ & 30 & $54.6 \pm 14.8$ & $37.2 \pm 48.8$ & $0.24 \pm 0.21$ & $0.07 \pm 0.12$ & $-0.17 \pm 0.19$ & $1 / 30(3.3 \%)$ & $26 / 30(86.7 \%)$ & $6 / 26(23.1 \%)$ & $37.7 \pm 42.8$ \\
\hline $\begin{array}{l}\text { Recurrent } \\
\text { erosions }\end{array}$ & 29 & $48.9 \pm 12.7$ & $30.9 \pm 38.9$ & $0.12 \pm 0.19$ & $0.05 \pm 0.16$ & $-0.07 \pm 0.18$ & $1 / 29(3.4 \%)$ & $23 / 29(79.3 \%)$ & $3 / 23(13.0 \%)$ & $9.7 \pm 1.5$ \\
\hline$P$-value & & 0.16 & 0.67 & 0.01 & 0.28 & 0.01 & 0.98 & 0.45 & 0.36 & 0.33 \\
\hline
\end{tabular}

Note: *Worse BCVA by $\geq 2$ lines (0.2 LogMAR).

Abbreviation: BCVA, best-corrected visual acuity.

and slit-lamp biomicroscopy before surgery. A total of 25 males and 26 females were included. The average age was 52 years, range (31-84 years). Of the 58 eyes, 29 (50\%) had recurrent erosions; 30 (52\%) had visual symptoms, including glare, photophobia, blur, or image distortion; and 4 (7\%) had a combination of erosions and visual symptoms. Patients were followed up for a minimum of 3 months postoperatively, with a range of follow-up from 3 to 170 months. A total of 32 eyes had at least 1 year of follow-up, and a total of 11 eyes had at least 5 years of follow-up.

\section{Visual acuity}

For patients presenting with symptoms of visual disturbances, the average visual acuity preoperatively was $~ 20 / 32$ (LogMAR $0.24 \pm$ SD 0.21). The mean final postoperative CDVA was $\sim 20 / 25$ (LogMAR $0.07 \pm$ SD 0.12; $P<0.0001$; Table 1 and Figure 1A). Of the 30 eyes in this cohort, $14(46.7 \%)$ gained $\geq 2$ lines of visual acuity, $8(26.7 \%)$ gained 1 line,
$7(23.3 \%)$ had the same CDVA, and $1(3.3 \%)$ lost 2 lines of visual acuity compared to preoperative measurement. CDVA gains were stable with no significant trend toward improving or worsening CDVA with longer follow-up (Figure 2A).

For patients presenting with symptoms of pain from recurrent erosions, the average visual acuity preoperatively was $\sim 20 / 25$ (LogMAR $0.12 \pm$ SD 0.19). The mean final postoperative CDVA was $\sim 20 / 20$ (LogMAR $0.05 \pm$ SD 0.16 ; $P=0.0785$; Figure 1B). Of the 29 eyes in this cohort, $5(17.2 \%)$ gained $\geq 2$ lines of visual acuity, $8(27.6 \%)$ had the same CDVA, $6(20.7 \%)$ lost 1 line, and 1 (3.4\%) lost 2 lines of visual acuity compared to preoperative measurement. CDVA gains were stable with no significant trend toward improving or worsening CDVA with longer follow-up (Figure 2B).

There was no statistically significant difference in postoperative CDVA between the 2 groups of patients. However, patients presenting with symptoms of visual disturbances did have worse preoperative CDVA compared to patients
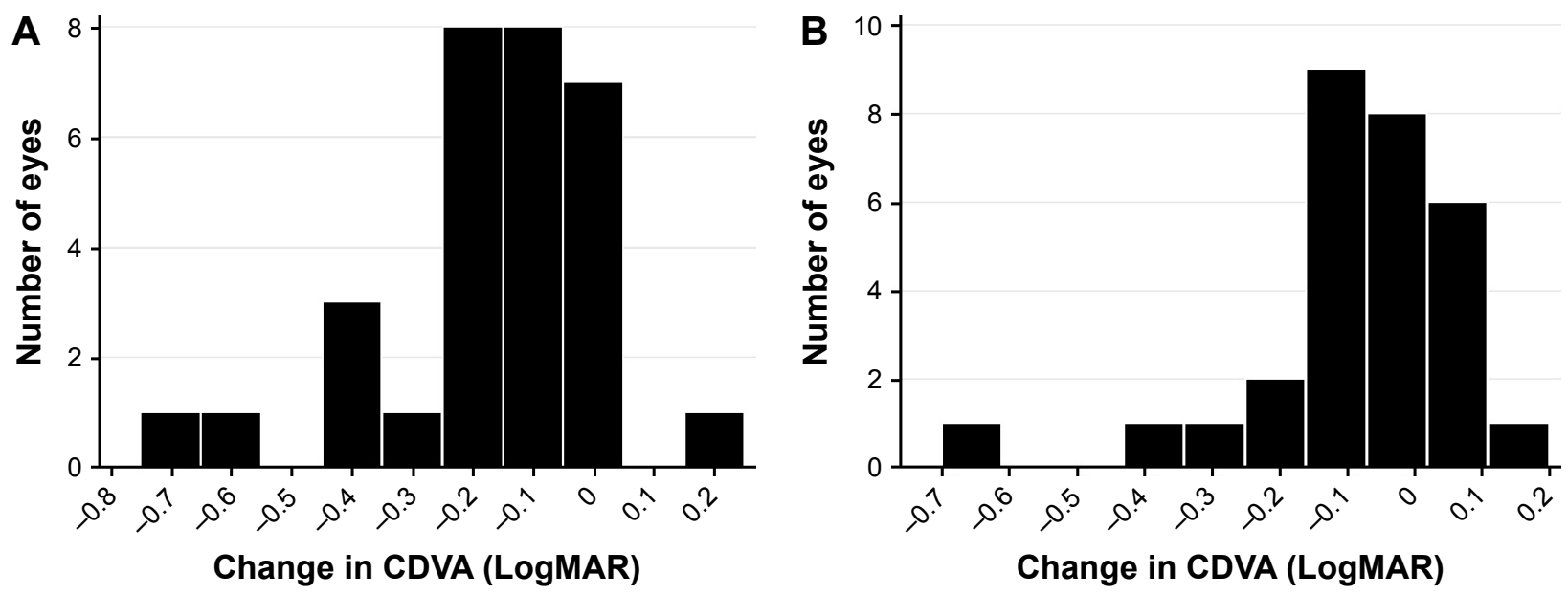

Figure I Graphs representing CDVA outcomes in eyes with EBMD refractory to medical treatment that were treated with PTK for (A) visual disturbances and (B) recurrent erosions.

Notes: The horizontal axis represents the change in CDVA in LogMAR (0.I LogMAR $=\sim$ I Snellen acuity line) between preoperative and postoperative measurements, with negative values corresponding to improved CDVA.

Abbreviations: CDVA, corrected distance visual acuity; EBMD, epithelial basement membrane dystrophy; PTK, phototherapeutic keratectomy. 


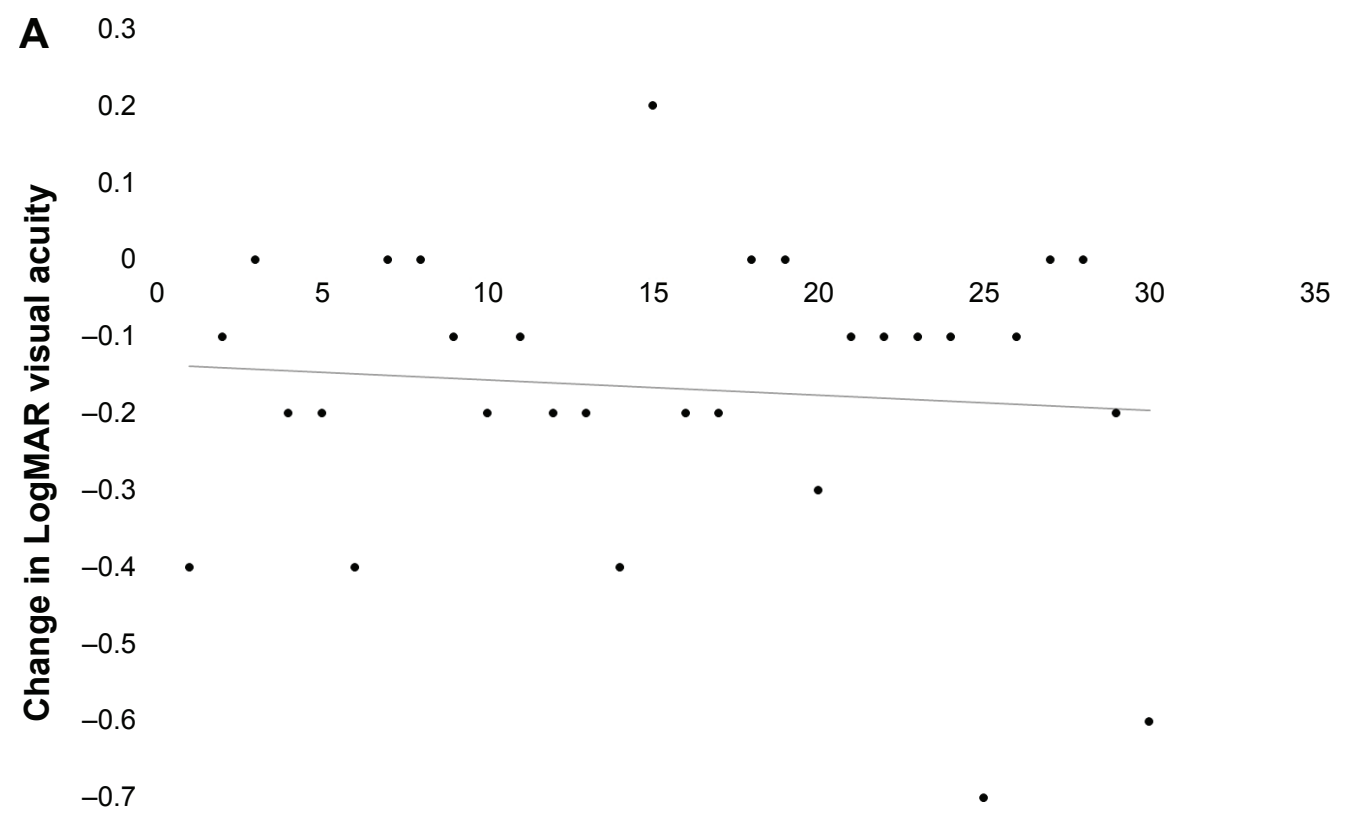

$-0.8 \quad$ Follow-up time (months)

B 0.3

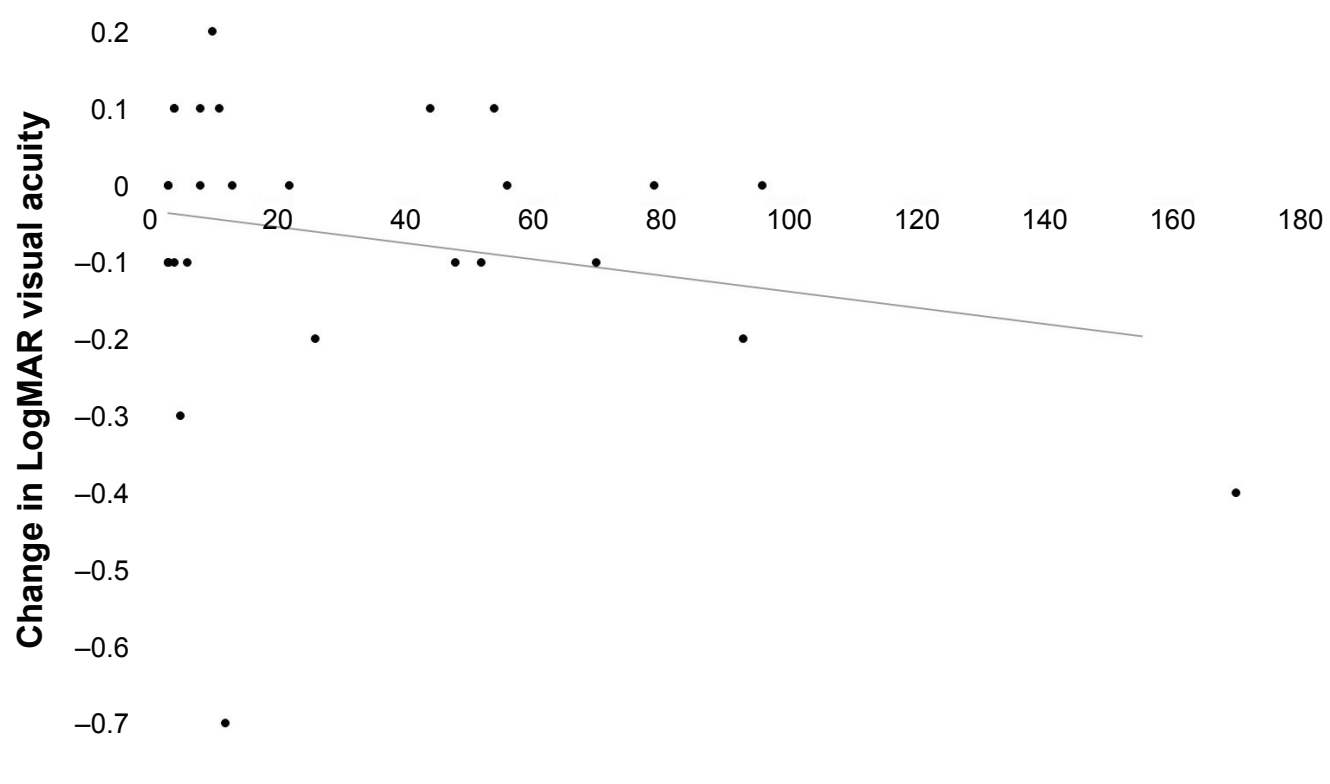

$-0.8$

Follow-up time (months)

Figure 2 Scatter plots of eyes with EBMD refractory to medical treatment that were treated with PTK for $(\mathbf{A})$ visual disturbances and $(\mathbf{B})$ recurrent erosions. Notes: Points represent change in CDVA in LogMAR plotted against time. Neither group $(\mathbf{A})(r=0.0903, P=0.636)$ or $(\mathbf{B})(r=-0.2356, P=0.22)$ show a significant trend, indicating stable visual acuity over time for both groups.

Abbreviations: EBMD, epithelial basement membrane dystrophy; PTK, phototherapeutic keratectomy; CDVA, corrected distance visual acuity.

presenting with recurrent erosions (LogMAR 0.24 vs 0.05 , $P=0.01)$ and also experienced a greater improvement in CDVA postoperatively $(-0.17$ vs $-0.07, P=0.01)$. Both groups of patients had similar rates of significant loss of visual acuity ( $>2$ lines; $3.3 \%$ vs $3.4 \%, P=0.98$ ).

\section{Refractive error}

For patients presenting with symptoms of visual disturbances, there was no statistically significant difference in preoperative versus postoperative manifest refraction spherical equivalent $(+0.25 \mathrm{D} \mathrm{SD} \pm 1.82, P=0.65)$. 
Similarly, for patients presenting with symptoms of pain from recurrent erosions, there was no statistically significant difference in preoperative versus postoperative manifest refraction spherical equivalent $(+0.26 \mathrm{D}$ SD \pm $1.44, P=0.63)$.

\section{Treatment response, recurrence, and retreatment}

For patients presenting with symptoms of visual disturbances, 26 out of 30 eyes (86.7\%) experienced initial resolution of symptoms following PTK. Of these 26 eyes, 6 eyes (23.1\%) experienced a recurrence of symptoms at an average follow-up time of 37.7 months postoperatively (range 4-105 months, SD 42.8). Among these eyes, $3(50 \%)$ had recurrence of visual disturbances within the first year postoperatively, 5 (83.3\%) had improved CDVA compared to preoperative values, and $1(16.7 \%)$ had unchanged CDVA compared to preoperative values. A total of 6 out of 30 eyes $(20.0 \%)$ demonstrated morphologic recurrence of EBMD findings on postoperative slit-lamp examination. However, there was no significant difference in mean CDVA between eyes with and without morphologic recurrence $(P=0.7734)$, and none of these eyes underwent a repeat treatment with PTK. The probability of being recurrence free from visual disturbances at 5 years postoperatively for eyes that had an initial treatment response to PTK was estimated at $83.0 \%$ (95\% confidence interval [CI] $68.7 \%-97.0 \%$ ) by the Kaplan-Meier analysis (Figure 3A).

For patients presenting with symptoms of pain from recurrent erosions, 23 out of 29 eyes (79.3\%) experienced initial resolution of symptoms following PTK. Of these 23 eyes,
3 eyes (13.0\%) experienced a recurrence of symptoms at an average follow-up time of 9.7 months postoperatively (range 8-11 months, SD 1.5). All 3 of these eyes had recurrence of painful erosions within the first year postoperatively. A total of 5 out of 29 eyes (17.2\%) demonstrated morphologic recurrence of EBMD findings on postoperative slit-lamp examination. Of these, only 1 eye (20\%) reported symptomatic recurrence of painful erosions. Of the 6 eyes that did not respond initially to PTK, 2 eyes underwent repeat PTK, with 1 of these eyes reporting improved but persistent symptoms at the final follow-up and 1 eye reporting no further symptoms at the final follow-up. Of the 3 eyes experiencing recurrence of painful erosions, 1 underwent repeat PTK with no further symptoms at the final follow-up. The probability of being recurrence free for symptomatic erosions at 5 years postoperatively for eyes that had an initial treatment response to PTK was estimated at $88.0 \%$ (95\% CI $65.3 \%-96.6 \%)$ by the Kaplan-Meier analysis (Figure 3B).

When comparing between the 2 groups, there were no statistically significant differences in rates of primary treatment response, rates of symptomatic recurrence, or time to recurrence.

\section{Complications}

Postoperatively all corneas reepithelialized within 14 days and $91 \%$ within 7 days, and no infections occurred. For patients presenting with symptoms of visual disturbances, the 1 eye (3.3\%) with reduced CDVA postoperatively had a final CDVA of 20/50 (LogMAR 0.4) and was noted to have corneal edema and stromal haze that had not been present
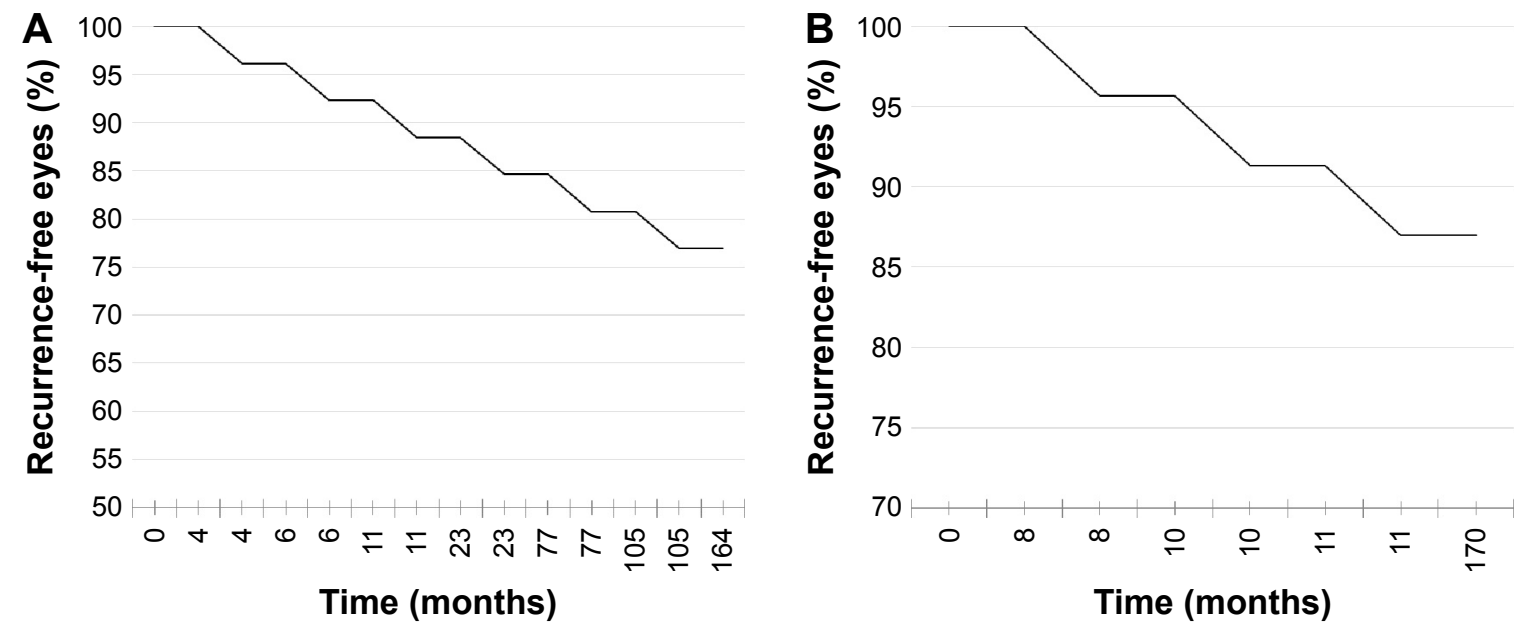

Figure 3 Kaplan-Meier estimate showing the cumulative probability of symptomatic recurrence for $(\mathbf{A})$ visual disturbances and (B) erosions after initial treatment response to PTK for EBMD refractory to medical treatment.

Notes: The probability of being recurrence free after initial treatment response for visual disturbances $(\mathbf{A})$ and erosions (B) at 5 years postoperatively was estimated at 83.0\% (95\% Cl 68.7\%-97.0\%) and 88.0\% (95\% Cl 65.3\%-96.6\%), respectively.

Abbreviations: PTK, phototherapeutic keratectomy; EBMD, epithelial basement membrane dystrophy; Cl, confidence interval. 
preoperatively. For patients presenting with symptoms of recurrent erosions, the 7 eyes (24\%) with reduced CDVA postoperatively had a mean final CDVA of $\sim 20 / 32$ (LogMAR $0.24 \pm$ SD 0.20). Of these eyes, 4 had no new ocular findings and clear corneas at the final slit-lamp examination and a mean final CDVA of 20/25 (LogMAR 0.1 \pm SD 0.08). The remaining 3 eyes had either guttata, stromal edema, or dots on the final slit-lamp examination, and a mean final CDVA of $\sim 20 / 50$ ( $\log M A R 0.43 \pm \mathrm{SD} 0.12)$. Of the 58 eyes examined, 9 eyes (15.5\%) showed new or progressed evidence of stromal haze or corneal opacity after PTK. There was no statistically significant difference in postoperative CDVA between eyes with and without new or progressed stromal haze or corneal opacity. Among eyes with new or progressed stromal haze, only $1(11.1 \%)$ demonstrated reduced CDVA by $>2$ lines, with $5(55.6 \%)$ showing improved CDVA and $3(33.3 \%)$ showing no change in CDVA.

\section{Discussion}

Our study of 58 PTK-treated eyes with EBMD is, to our knowledge, the largest series described thus far for EBMD treated with PTK. The results of our study support previous findings that PTK is effective in treating both the recurrent erosions and visual disturbances that result from EBMD. ${ }^{2,8,12-14}$ Moreover, this study has demonstrated that improvement in visual acuity and epithelial integrity is stable in long-term follow-up.

In comparing outcomes between these 2 groups, we found similar rates of primary treatment response, with the majority of patients responding to treatment and the majority of responders having sustained relief from symptoms on longterm follow-up. Although recurrence of erosions tended to present earlier than recurrence of visual disturbances, there was no statistically significant difference in time to recurrence between the 2 groups.

Overall, we found PTK for EBMD to be a safe procedure with similarly low complication rates between the 2 groups. The only significant difference in the complication rate between the 2 groups was the greater proportion of patients with reduced postoperative CDVA in patients presenting with recurrent erosions. We hypothesize that this is related to the better preoperative CDVA for this group at baseline and thus higher probability of worse CDVA on repeat testing with expected fluctuations in performance. In support of this hypothesis, there was no significant difference in postoperative CDVA between the 2 groups of patients.

Other commonly practiced methods of surgically treating EBMD include epithelial debridement alone or epithelial debridement with anterior stromal puncture or diamonddusted burr polishing of Bowman's layer. Our outcomes appear comparable to the reported outcomes for these treatment modalities.

Itty et $\mathrm{al}^{15}$ reported a study of 74 eyes treated with epithelial debridement with and without anterior stromal puncture. Although both eyes with visual disturbances and painful erosions were included, there was no separate analysis performed for these 2 groups. They found that at an average 33 months follow-up, there was a $24 \%$ morphologic recurrence rate that projected to $44.7 \%$ by 5 years. However, they were unable to determine the symptomatic effect of this morphologic recurrence. We found a similar rate of morphologic recurrence at the final follow-up (20.0\% for patients with visual disturbances and $17.2 \%$ for patients with painful erosions), but many of these patients did not experience recurrent symptoms. All eyes in that study had same or improved CDVA at follow-up. Although our study had a larger proportion of patients with worse postoperative CDVA, this was seen primarily in our patients with painful erosions, which comprised only $18 \%$ of eyes in the study by Itty et al, while comprising $49.2 \%$ of our study population.

Aldave et $\mathrm{al}^{16}$ found that the use of epithelial debridement and diamond-dusted burr polishing of Bowman's layer for recurrent corneal erosions and visually significant epithelial irregularity associated with EBMD and corneal trauma resulted in improved visual acuity and resolved recurrent erosions at an average 18.9 months follow-up in $96 \%$ of 25 eyes, which compared favorably to our primary treatment response rate of $79.3 \%$ for recurrent erosions. They found that $12 / 47$ (25.5\%) eyes developed new mild central subepithelial haze but that this haze was not associated with decreased vision. This was similar to our rate of new corneal haze postoperatively (15.5\%). In a follow-up study by the same group, epithelial debridement and diamond-dusted burr polishing were found to result in no recurrent erosions in 95\% of 37 eyes with a mean of 33.2 months of follow-up. ${ }^{17}$ In all 22 eyes treated for visual disturbances with $>3$ months of follow-up, there was no morphologic recurrence of EBMD and recurrence of symptomatic visual disturbance. Soong et $\mathrm{al}^{18}$ evaluated the use of diamond-dusted burr polishing of Bowman's layer in the setting of recurrent erosions due to both EBMD and no corneal dystrophy. Over an average follow-up of 12.3 months, they found a $6 \%$ rate of recurrent erosions and concluded that diamond-dusted burr polishing was a safe and less costly alternative to PTK for the treatment of recurrent erosions due to EBMD. 
Sridhar et $\mathrm{al}^{12}$ compared the outcomes of PTK versus diamond-dusted burr polishing of Bowman's layer specifically for recurrent erosions in EBMD. In 15 eyes, Bowman's membrane was ablated with PTK, and 27 eyes underwent diamond-dusted burr to polish Bowman's membrane in the area of the epithelial defect. They reported a higher incidence of corneal haze in the PTK group compared to that in the diamond burr group, 5 eyes (35.7\%) compared to 7 eyes (25.9\%), respectively. A higher rate of recurrent erosions was reported in the PTK group than in the diamond burr group, 4 eyes (26.7\%) compared to 3 eyes (11.1\%), respectively. Visual acuity outcomes were slightly better in the PTK group than those in the diamond burr group. They found no statistical difference between the groups in terms of visual outcome, corneal haze, or recurrence. Our series reports a lower rate of corneal haze, (15.5\%), with no statistical difference in CDVA between the groups with and without new corneal haze. We report a lower recurrence rate for erosions $(13.0 \%)$ but a larger proportion of eyes with reduced visual acuity (24.1\%). Notably, Sridhar et al did not explicitly report their primary treatment response rate, whereas our recurrence rate for erosions is specifically for patients who experienced a primary treatment response.

Previous studies reporting on the outcomes of PTK for EBMD show similar results in visual improvement with differences in the recurrence rate that may be accounted for by differences in the population and type of recurrence. Orndahl and Fagerholm ${ }^{8}$ presented on their outcomes of EBMD treated with PTK. Their technique was similar, and of 17 eyes with longstanding visual reduction, 14 (82.4\%) had increased visual acuity by $\geq 2$ lines at a mean follow-up interval of 30 months. No morphologic recurrence was seen in the treatment zone of any of the 24 eyes, with 1 eye out of 10 experiencing recurrence of recurrent corneal erosions. ${ }^{2,8}$ Pogorelov et a ${ }^{11}$ reported similar results using a similar technique demonstrating no recurrences of erosions in 15 eyes following PTK at a mean follow-up of 4.8 years. In 12 cases of EBMD, Dinh et a $1^{13}$ found a $42 \%$ recurrence rate of any type such as recurrent erosions or decreased vision plus slit-lamp morphological findings within the first year. Germundsson et al ${ }^{14}$ reported a $46 \%$ total recurrence rate and $13 \%$ symptomatic recurrence rate for either visual disturbances or erosions in 51 eyes at an average 43 months of follow-up.

There are important limitations to the present study. First, the retrospective study design did not allow for prospective ascertainment of recurrence. This also led to heterogeneous follow-up times and thus nonuniform data. Future studies assessing efficacy of treatment for EBMD may benefit from prospective data collection to avoid these limitations.

\section{Conclusion}

Our results have demonstrated that both visual disturbances and painful erosions associated with EBMD can be expected to respond to PTK in the majority of cases, and for those cases that show a treatment response, relief from symptoms is maintained over long-term follow-up. Prior studies have shown good efficacy and safety for less costly alternatives, including epithelial debridement, anterior stromal micropuncture, and diamond-dusted burr polishing. Thus, despite the positive outcomes we report here for PTK in treating EBMD, ophthalmologists may want to consider initial treatment with more cost-effective treatment modalities prior to PTK.

EBMD is a heterogenous group of dystrophies leading to the classic EBMD changes. ${ }^{3,19}$ PTK may not correct the underlying pathology in some forms of EBMD, perhaps leading to a subgroup of patients with either no primary treatment response or early recurrence after PTK. More detailed studies exploring the clinical-molecular correlations behind EBMD are needed to better understand and predict both treatment response and recurrence. In the meantime, the results of this study may better inform ophthalmologists and their patients regarding the likelihood of treatment response, recurrence, and complications for PTK in the setting of EBMD.

\section{Disclosure}

Doctor Lee and Doctor Lam have no relevant financial interests to disclose. Doctor Manche owns equity in Calhoun Vision, Inc., Seros Medical, LLC., and Veralas, Inc. He is a consultant for Allergan, Abbott Medical Optics, Avellino Laboratories, Carl Zeiss, and Guidepost Global. He holds patents with Seros Medical. The authors report no other conflicts of interest in this work.

\section{References}

1. Zuckerman SJ, Aquavella JV, Park SB. Analysis of the efficacy and safety of excimer laser PTK in the treatment of corneal disease. Cornea. 1996; 15(1):9-14.

2. Fagerholm P. Phototherapeutic keratectomy: 12 years of experience. Acta Ophthalmol Scand. 2003;81(1):19-32.

3. Waring GO 3rd, Rodrigues MM, Laibson PR. Corneal dystrophies. I. Dystrophies of the epithelium, Bowman's layer and stroma. Surv Ophthalmol. 1978;23(2):71-122.

4. Werblin TP, Hirst LW, Stark WJ, Maumenee IH. Prevalence of mapdot-fingerprint changes in the cornea. Br J Ophthalmol. 1981;65(6): 401-409.

5. Ewald M, Hammersmith KM. Review of diagnosis and management of recurrent erosion syndrome. Curr Opin Ophthalmol. 2009; 20(4):287-291. 
6. Laibson PR. Recurrent corneal erosions and epithelial basement membrane dystrophy. Eye Contact Lens. 2010;36(5):315-317.

7. Reidy JJ, Paulus MP, Gona S. Recurrent erosions of the cornea: epidemiology and treatment. Cornea. 2000;19(6):767-771.

8. Orndahl MJ, Fagerholm PP. Phototherapeutic keratectomy for mapdot-fingerprint corneal dystrophy. Cornea. 1998;17(6):595-599.

9. Geggel HS. Successful treatment of recurrent corneal erosion with Nd:YAG anterior stromal puncture. Am J Ophthalmol. 1990;110(4): 404-407.

10. Tzelikis PF, Rapuano CJ, Hammersmith KM, Laibson PR, Cohen EJ. Diamond burr treatment of poor vision from anterior basement membrane dystrophy. Am J Ophthalmol. 2005;140(2):308-310.

11. Pogorelov P, Langenbucher A, Kruse F, Seitz B. Long-term results of phototherapeutic keratectomy for corneal map-dot-fingerprint dystrophy (Cogan-Guerry). Cornea. 2006;25(7):774-777.

12. Sridhar MS, Rapuano CJ, Cosar CB, Cohen EJ, Laibson PR. Phototherapeutic keratectomy versus diamond burr polishing of Bowman's membrane in the treatment of recurrent corneal erosions associated with anterior basement membrane dystrophy. Ophthalmology. 2002;109(4):674-679.

13. Dinh R, Rapuano CJ, Cohen EJ, Laibson PR. Recurrence of corneal dystrophy after excimer laser phototherapeutic keratectomy. Ophthalmology. 1999;106(8):1490-1497.
14. Germundsson J, Fagerholm P, Lagali N. Clinical outcome and recurrence of epithelial basement membrane dystrophy after phototherapeutic keratectomy a cross-sectional study. Ophthalmology. 2011; 118(3):515-522.

15. Itty S, Hamilton SS, Baratz KH, Diehl NN, Maguire LJ. Outcomes of epithelial debridement for anterior basement membrane dystrophy. Am J Ophthalmol. 2007;144(2):217-221.

16. Aldave AJ, Kamal KM, Vo RC, Yu F. Epithelial débridement and Bowman's layer polishing for visually significant epithelial irregularity and recurrent corneal erosions. Cornea. 2009;28(10):1085-1090.

17. Vo RC, Chen JL, Sanchez PJ, Yu F, Aldave AJ. Long-term outcomes of epithelial debridement and diamond burr polishing for corneal epithelial irregularity and recurrent corneal erosion. Cornea. 2015; 34(10):1259-1265.

18. Soong HK, Farjo Q, Meyer RF, Sugar A. Diamond burr superficial keratectomy for recurrent corneal erosions. $\mathrm{Br} J$ Ophthalmol. 2002;86(3):296-298.

19. Fogle JA, Kenyon KR, Stark WJ, Green WR. Defective epithelial adhesion in anterior corneal dystrophies. Am J Ophthalmol. 1975; 79(6):925-940.
Clinical Ophthalmology

\section{Publish your work in this journal}

Clinical Ophthalmology is an international, peer-reviewed journal covering all subspecialties within ophthalmology. Key topics include: Optometry; Visual science; Pharmacology and drug therapy in eye diseases; Basic Sciences; Primary and Secondary eye care; Patient Safety and Quality of Care Improvements. This journal is indexed on

\section{Dovepress}

PubMed Central and CAS, and is the official journal of The Society of Clinical Ophthalmology (SCO). The manuscript management system is completely online and includes a very quick and fair peer-review system, which is all easy to use. Visit http://www.dovepress.com/ testimonials.php to read real quotes from published authors. 